\title{
On small deformations of paracomplex manifolds
}

\author{
Costantino Medori and Adriano Tomassini*
}

\begin{abstract}
A paracomplex structure on a manifold $M$ is an endomorphism $K$ of the tangent bundle $T M$ such that $K^{2}=I$, whose \pm 1 -eigenspaces have the same dimension and are involutive. By using the theory of differential graded Lie algebras, we describe small deformations of paracomplex structures. We also compute the space of invariant small deformations of 4-dimensional nilmanifolds endowed with a fixed paracomplex structure.
\end{abstract}

Mathematics Subject Classification (2010). 53C15, 32G07.

Keywords. Paracomplex structure, deformation, differential graded Lie algebra, nilmanifold.

\section{Introduction}

Let $M$ be a $2 n$-dimensional manifold. An almost paracomplex structure on $M$ is an endomorphism $K$ of the tangent bundle $T M$ of $M$ such that $K^{2}=I_{T M}$ and the two distributions

$$
T^{ \pm} M=\{X \pm K X \mid X \in \Gamma(M, T M)\}
$$

have the same rank (see [11]). An almost paracomplex structure $K$ is said to be a paracomplex structure if these two distributions are involutive (this condition is equivalent to the vanishing of the torsion tensor of $K$ ). In fact, a paracomplex structure on a manifold can be given by a pair of transverse foliations of the same dimension. If the paracomplex manifold $(M, K)$ admits a pseudo-Riemannian metric $g$ such that $g(K X, K Y)=-g(X, Y)$ and the fundamental form $\omega(X, Y)=g(X, K Y)$ is closed, then the datum $(K, g, \omega)$ is a para-Kähler structure on $M$. It turns out that a para-Kähler structure on $M$ gives rise to a pair of involutive Lagrangian distributions $T^{-} M$ and $T^{+} M$ such that $T M=T^{+} M \oplus T^{-} M$, called bi-Lagrangian structure (see [3]). Conversely, starting from a symplectic manifold $(M, \omega)$ whose tangent bundle $T M$ is the direct sum of two Lagrangian involutive distributions $T^{ \pm} M$, one can define a para-Kähler structure on $M$, setting

$$
\left.K\right|_{T^{ \pm}{ }_{M}}= \pm I \quad \text { and } \quad g(X, Y)=\omega(X, K Y) .
$$

\footnotetext{
* This work was supported by the Project M.I.U.R. "Geometric Properties of Real and Complex Manifolds" and by G.N.S.A.G.A. of I.N.d.A.M.
} 
In this paper we study small deformations of a paracomplex structure on a compact manifold. In order to do this, as in the theory of small deformations of complex structures (see [13]) or $C R$-structures (see [7]), we introduce a differential graded Lie algebra $\left(\mathcal{A},[[]],, \bar{\partial}_{K}\right)$ (see Section 5). Then it turns out that small paracomplex deformations of $K$ are parametrized by degree 1 elements of $\mathcal{A}=\bigoplus_{p \in \mathbb{Z}} \mathcal{A}_{p}$ satisfying the Maurer-Cartan equation. First of all we show that every almost paracomplex structure $\widehat{K}$ close to $K$ can be written as

$$
\widehat{K}=(I+L) K(I+L)^{-1},
$$

where $L \in \operatorname{End}(T M)$ anticommutes with $K$ and $\operatorname{det}(I+L) \neq 0$ (see Proposition 3.1). Then we prove the following (see Theorem 4.2).

Theorem. Let $(M, K)$ be a compact paracomplex manifold. Then the map between

$$
\left\{L \in \Gamma(M, \operatorname{End}(T M)) \mid L K+K L=0, \bar{\partial}_{K} L+\frac{1}{2}[[L, L]]=0, \operatorname{det}(I+L) \neq 0\right\}
$$

and

given by

$$
\left\{\widehat{K} \in \Gamma(M, \operatorname{End}(T M)) \mid \widehat{K}^{2}=I, N_{\widehat{K}}=0\right\},
$$

$$
L \mapsto \widehat{K}=(I+L) K(I+L)^{-1},
$$

is a bijection between a neighborhood of $0 \in \Gamma(M, \operatorname{End}(T M))$ and a neighborhood of $K$.

The paper is organized as follows. In Section 1 we recall some general definitions and results on paracomplex geometry.

Section 2 is devoted to the construction of the differential graded Lie algebra governing the small deformation theory of a paracomplex structure. In particular, we give explicit formulas for the bracket $[[]$,$] and for the \bar{\partial}_{K}$-operator without using local coordinates.

In Section 3 we consider curves of paracomplex structures through a paracomplex structure $K$, characterizing almost paracomplex structures close to $K$ (see Proposition 3.1) and finding obstructions in order that an endomorphism $L$ anticommuting with $K$ gives rise to a curve of paracomplex structures (see Corollary 3.3).

In Section 4 we give the proof of the main result (Theorem 4.2). A key tool is given by Proposition 4.1.

Finally, in the last section we apply the results previously obtained to describe small deformations on 4-dimensional nilmanifolds endowed with a fixed paracomplex structure. By classification, there are two (non-abelian) nilpotent Lie algebras, whose (compact) nilmanifolds associated are either primary Kodaira-Thurston surfaces or have first Betti number $b_{1}=2$. We fix a paracomplex structure on these two nilmanifolds and we determine the invariant small paracomplex deformations of them. We show that such spaces depend on six and four real parameters, respectively. 
It has to be remarked that a nilmanifold with $b_{1}=2$ cannot have any complex structure (see Remark 5.2). For small deformations of left invariant complex structures on nilmanifolds see [4] in the case of abelian complex structures and [15] in the general case. We also construct a family of para-Kähler structures on the primary Kodaira-Thurston surface.

The authors would like to thank Vicente Cortés and the anonymous referee for useful comments.

\section{Generalities on paracomplex manifolds}

We recall some basic definitions of paracomplex geometry (see e.g [6], [5], [1]). Let $V$ be a $2 n$-dimensional real vector space. A paracomplex structure on $V$ is an endomorphism $K: V \rightarrow V$ such that

i) $K^{2}=I_{V}$,

ii) the eigenspaces $V^{+}, V^{-}$of $K$ with eigenvalues $1,-1$, respectively, have the same dimension.

The pair $(V, K)$ will be called a paracomplex vector space.

Definition 1.1. An almost paracomplex structure on a $2 n$-dimensional manifold $M$ is the datum of a section $K$ of $\operatorname{End}(T M)$ such that

i) $K^{2}=I_{T M}$,

ii) the two eigendistributions $T^{ \pm} M:=\operatorname{ker}(I \mp K)$ have the same rank.

A almost paracomplex structure $K$ is said to be integrable if the distributions $T^{ \pm} M$ are involutive. In such a case $K$ is called a paracomplex structure. A manifold $M$ endowed with an (almost) paracomplex structure $K$ is called an (almost) paracomplex manifold.

The Nijenhuis tensor $N_{K}$ of an almost paracomplex structure $K$ is the $(1,1)$-tensor field defined by

$$
N_{K}(X, Y)=[X, Y]+[K X, K Y]-K[K X, Y]-K[X, K Y]
$$

for every pair of vector fields $X, Y$ on $M$. As in the complex case, an almost paracomplex structure $K$ is integrable if and only if $N_{K}=0$ (see e.g. [5]).

A basic example of a paracomplex manifold is given by $\mathbb{R}^{2 n}$, with coordinates $\left(x_{1}, \ldots, x_{n}, y_{1}, \ldots, y_{n}\right)$, endowed with the standard paracomplex structure $K_{n}$ defined as

$$
K_{n}\left(\frac{\partial}{\partial x_{j}}\right)=\frac{\partial}{\partial y_{j}}, \quad K_{n}\left(\frac{\partial}{\partial y_{j}}\right)=\frac{\partial}{\partial x_{j}}, \quad j=1, \ldots, n .
$$

We recall that the set of paracomplex numbers

$$
\mathcal{C}=\left\{z=x+e y \mid x, y \in \mathbb{R}, e^{2}=1\right\}
$$


is endowed with the natural operations of sum, (distributive) product and multiplication by a real number. Then $\mathcal{C}$ is a ring and a vector space over $\mathbb{R}$. Note that $\mathcal{C}$ is not an integral domain and $z=x+e y \in \mathcal{C}$ is invertible if and only if $x^{2}-y^{2} \neq 0$. Let $(M, K)$ be an almost para-complex manifold. Set $T^{\mathcal{C}} M=\mathcal{C} \otimes_{\mathbb{R}} T M$ and extend the endomorphism $K$ to a $\mathcal{C}$-linear endomorphism of $T^{\mathcal{C}} M$. Then, for every $p \in M$,

$$
T_{p}^{e} M=T_{p}^{1,0} M \oplus T_{p}^{0,1} M,
$$

where

$$
\begin{aligned}
& T_{p}^{1,0} M=\left\{Z \in T_{p}^{e} M \mid K Z=e Z\right\}=\left\{X+e K X \mid X \in T_{p} M\right\}, \\
& T_{p}^{0,1} M=\left\{Z \in T_{p}^{\complement} M \mid K Z=-e Z\right\}=\left\{X-e K X \mid X \in T_{p} M\right\}
\end{aligned}
$$

are the "eigenspaces" of $K$ with "eigenvalues" $\pm e$, being $T_{p}^{\varphi} M$ a $\ell$-module.

For every $Z=X+e Y \in T_{p}^{e} M$ denote, as usual, the conjugate of $Z$ by $\bar{Z}=$ $X-e Y$. Then $T_{p}^{0,1} M=\bar{T}_{p}^{1,0} M$.

The almost para-complex structure $K$ acts naturally on $\left(T^{\mathcal{C}}\right)^{*} M$ by

$$
K^{*} \alpha(X)=\alpha(K X) .
$$

We have a decomposition

$$
\left(T^{\mathcal{C}}\right)^{*} M=\bigwedge_{K}^{1,0}(M) \oplus \bigwedge_{K}^{0,1}(M),
$$

where

$$
\begin{aligned}
& \bigwedge_{K}^{1,0}(M):=\left\{\alpha+e K^{*} \alpha \mid \alpha \in T^{*} M\right\}, \\
& \bigwedge_{K}^{0,1}(M):=\left\{\alpha-e K^{*} \alpha \mid \alpha \in T^{*} M\right\}
\end{aligned}
$$

are eigenspaces for $K^{*}$ with eigenvalues $\pm e$. The last decomposition induces a splitting of the bundle $\wedge^{r}\left(T^{\mathcal{E}}\right)^{*} M$ of para-complex $r$-forms on $(M, K)$ given by

$$
\wedge^{r}\left(T^{\mathcal{e}}\right)^{*} M=\bigoplus_{p+q=r} \bigwedge_{K}^{p, q}(M) .
$$

The sections of $\bigwedge_{K}^{p, q}(M)$ are the $(p, q)$-forms on the almost paracomplex manifold $(M, K)$.

A para-Hermitian metric on an almost paracomplex manifold $(M, K)$ is the datum of pseudo-Riemannian metric $g$ of signature $(n, n)$ such that

$$
g(K X, K Y)=-g(X, Y)
$$

for every pair of vector fields $X, Y$ in $M$. The fundamental form of the para-Hermitian $g$ is the 2 -form $\omega$ defined by

$$
\omega(X, Y)=g(X, K Y) .
$$

The para-Hermitian metric $g$ is said to be para-Kähler if $N_{K}=0$ and $d \omega=0$. 


\section{Paracomplex structures and DGLA}

Let $(M, K)$ be an $n$-dimensional paracomplex manifold. We are going to define the differential graded Lie algebra, shortly DGLA, governing the small deformations of $K$ (for a general theory of deformations see e.g. [9]). Let $\Omega_{K}^{0, p}(M)$ be the space of sections of the bundle of $(0, p)$-forms on $(M, K)$. Denote by $\Gamma\left(M, \wedge_{K}^{0, p}(M) \otimes\right.$ $\left.T^{1,0} M\right)$ the space of sections of the vector bundle $\bigwedge_{K}^{0, p}(M) \otimes T^{1,0}(M)$. Set

$$
\mathcal{A}_{p}= \begin{cases}\Gamma\left(M, \bigwedge_{K}^{0, p}(M) \otimes T^{1,0} M\right) & \text { if } 0 \leq p \leq n, \\ 0 & \text { otherwise }\end{cases}
$$

and define

$$
\mathcal{A}=\bigoplus_{p \in \mathbb{Z}} \mathcal{A}_{p}
$$

Note that the real vector space $\mathcal{A}$ is a module over the paracomplex numbers. Now we introduce a bracket $[[]$,$] and a \bar{\partial}_{K}$-operator on the $\mathcal{C}$-module $\mathcal{A}$. Namely,

Definition 2.1. The bracket

$$
[[,]]: \mathcal{A}_{p} \times \mathcal{A}_{q} \rightarrow \mathcal{A}_{p+q}
$$

is defined in the following way:

- For every pair $Z, W \in \mathcal{A}_{0}$ set

$$
[[Z, W]]=[Z, W],
$$

where $[$,$] is the usual bracket between complex vector fields.$

- For every $\varphi \in \mathcal{A}_{1}$ and $Z \in \mathcal{A}_{0}$, define $[[\varphi, Z]]=-[[Z, \varphi]] \in \mathcal{A}_{1}$ as

$$
[[\varphi, Z]](\bar{W})=[\varphi(\bar{W}), Z]+\frac{1}{2} \varphi([Z, \bar{W}]-e K[Z, \bar{W}]) .
$$

- For every $\varphi \in \mathcal{A}_{1}$, define $[[\varphi, \varphi]] \in \mathcal{A}_{2}$ as

$$
[[\varphi, \varphi]](\bar{Z}, \bar{W})=2[\varphi(\bar{Z}), \varphi(\bar{W})]-2 \varphi([\varphi(\bar{Z}), \bar{W}]+[\bar{Z}, \varphi(\bar{W})]) .
$$

- For every $\varphi, \psi \in \mathcal{A}_{1}$, define $[[\varphi, \psi]] \in \mathcal{A}_{2}$ as

$$
[[\varphi, \psi]]=\frac{1}{2}([[(\varphi+\psi),(\varphi+\psi)]]-[[\varphi, \varphi]]-[[\psi, \psi]]) .
$$

- For every $\alpha \in \Omega_{K}^{0, p}(M), \beta \in \Omega_{K}^{0, q}(M), \varphi, \psi \in \mathcal{A}_{1}$, set

$$
\begin{gathered}
{[[\alpha \wedge \varphi, \beta \wedge \psi]]=(-1)^{q} \alpha \wedge \beta \wedge[[\varphi, \psi]]+(-1)^{p}\left(\boldsymbol{T}_{\psi} \alpha\right) \wedge \beta \wedge \varphi} \\
+\alpha \wedge\left(\mathrm{T}_{\varphi} \beta\right) \wedge \psi,
\end{gathered}
$$


where $\top_{\varphi}$ is the skew-symmetric derivation of degree 1 of $\Omega^{0, *}(M)$ such that, for every smooth function $f,\left(T_{\varphi} f\right)(\bar{Z})=\varphi(\bar{Z})(f)$, and, for every $\alpha \in \Omega_{K}^{0,1}(M)$,

$\left(\boldsymbol{\top}_{\varphi} \alpha\right)(\bar{Z}, \bar{W})=\varphi(\bar{Z})(\alpha(\bar{W}))-\varphi(\bar{W})(\alpha(\bar{Z}))-\alpha([\varphi(\bar{Z}), \bar{W}]+[\bar{Z}, \varphi(\bar{W})])$.

- Finally, extend [[, ]] by bilinearity to any pair of elements of $\mathcal{A}$.

Definition 2.2. The $\bar{\partial}_{K}$-operator is the map

$$
\bar{\partial}_{K}: \mathcal{A}_{p} \rightarrow \mathcal{A}_{p+1}
$$

defined by:

i) For $Z \in \mathcal{A}_{0}$, set

$$
\left(\bar{\partial}_{K} Z\right)(\bar{W})=\frac{1}{2}([\bar{W}, Z]+e K[\bar{W}, Z])
$$

for vector field $\bar{W}$ of type $(0,1)$.

ii) For $\varphi \in \mathcal{A}_{1}$, set

$$
\left(\bar{\partial}_{K} \varphi\right)(\bar{Z}, \bar{W})=\left(\bar{\partial}_{K} \varphi(\bar{W})\right) \bar{Z}-\left(\bar{\partial}_{K} \varphi(\bar{Z})\right) \bar{W}-\varphi([\bar{Z}, \bar{W}])
$$

for every pair of vector fields $\bar{Z}, \bar{W}$ of type $(0,1)$.

iii) Extend $\bar{\partial}_{K}$ to $\mathcal{A}_{p}$ by Leibniz rule, i.e.,

$$
\bar{\partial}_{K}(\alpha \wedge \varphi)=\bar{\partial}_{K} \alpha \wedge \varphi+(-1)^{\operatorname{deg}(\alpha)} \alpha \wedge \bar{\partial}_{K} \varphi
$$

for every $\alpha \in \Omega_{K}^{0, p}(M), \varphi \in \mathcal{A}_{1}$.

Note that, for any $\varphi \in \mathcal{A}_{p}, \psi \in \mathcal{A}_{q}, \rho \in \mathcal{A}_{r}$, it can be checked that

1) $[[\varphi, \psi]]=-(-1)^{\operatorname{deg}(\varphi) \operatorname{deg}(\psi)}[[\psi, \varphi]]$,

2) $[[\varphi,[[\psi, \rho]]]]=[[[[\varphi, \psi]], \rho]]+(-1)^{\operatorname{deg}(\varphi) \operatorname{deg}(\psi)}[[\psi,[[\varphi, \rho]]]]$,

3) $\bar{\partial}_{K}^{2}=0$,

4) $\bar{\partial}_{K}[[\varphi, \psi]]=\left[\left[\bar{\partial}_{K} \varphi, \psi\right]\right]+(-1)^{\operatorname{deg}(\varphi)}\left[\left[\varphi, \bar{\partial}_{K} \psi\right]\right]$.

Therefore, $\left(\mathcal{A},[[]],, \bar{\partial}_{K}\right)$ is a DGLA.

Now we note that the theory can be set in real terms. To this purpose, define

$$
\begin{aligned}
\mathcal{A}_{p}^{\mathbb{R}}=\left\{\varphi \in \Gamma\left(M, \bigwedge^{p}(M) \otimes T M\right) \mid\right. & \varphi\left(X_{1}, \ldots, K X_{j}, \ldots, X_{p}\right) \\
& \left.=-K \varphi\left(X_{1}, \ldots, X_{p}\right) \text { for all } j=1, \ldots, p\right\},
\end{aligned}
$$

e.g., $\mathcal{A}_{0}^{\mathbb{R}}$ is the space of smooth vector fields and

$$
\mathcal{A}_{1}^{\mathbb{R}}=\{L \in \operatorname{End}(T M) \mid L K+K L=0\} .
$$


Let $m: T M \rightarrow T^{1,0} M$ be defined as

$$
m(X)=\frac{1}{2}(X+e K X),
$$

with inverse $m^{-1}$ defined by $m^{-1}(R)=R+\bar{R}$.

Then every element $L \in \mathcal{A}_{p}^{\mathbb{R}}$ can be identified with $m(L) \in \mathcal{A}_{p}$ by setting

$$
m(L)=\frac{1}{2}(L+e K L) .
$$

In particular, for every pair of vector fields $X, Y$, we have

$$
\begin{aligned}
{[[X, Y]] } & =m^{-1}[[m(X), m(Y)]]=\frac{1}{2}([X, Y]+[K X, K Y]), \\
\left(\bar{\partial}_{K} X\right)(Y) & =\frac{1}{2}([Y, X]-K[K Y, X]),
\end{aligned}
$$

and, for every $L \in \mathcal{A}_{1}^{\mathbb{R}}$,

$$
\left(\bar{\partial}_{K} L\right)(X, Y)=\left(\bar{\partial}_{K} L Y\right)(X)-\left(\bar{\partial}_{K} L X\right)(Y)-L[[X, Y]],
$$

where we used the identification $\bar{\partial}_{K}=m^{-1} \circ \bar{\partial}_{K} \circ m$.

By formula (1), it follows immediately that

$$
\left(\bar{\partial}_{K} K X\right)(Y)=-\left(\bar{\partial}_{K} X\right)(K Y) .
$$

Finally, in a similar way, for every $L \in \mathcal{A}_{1}^{\mathbb{R}}$ we have

$$
[[L, L]](X, Y)=2[[L X, L Y]]+2 L\left(\left(\bar{\partial}_{K} X\right)(L Y)-\left(\bar{\partial}_{K} Y\right)(L X)\right) .
$$

Indeed, a direct computation yields

$$
\begin{aligned}
& {[[L, L]](X, Y)} \\
& =[L X, L Y]+[K L X, K L Y] \\
& \quad-\frac{1}{2}\{L([L X, Y]-[K L X, K Y])+K L([K L X, Y]-[L X, K Y])\} \\
& \quad-\frac{1}{2}\{L([X, L Y]-[K X, K L Y])+K L([X, K L Y]-[K X, L Y])\} .
\end{aligned}
$$

\section{Curves of paracomplex structures}

We consider the space of linear paracomplex structures on $\mathbb{R}^{2 n}$ :

$$
\mathcal{X}(n)=\left\{P \in \mathrm{GL}(2 n, \mathbb{R}) \mid P^{2}=I, \operatorname{tr}(P)=0\right\} .
$$

Let $K_{n}$ be the standard linear paracomplex structure on $\mathbb{R}^{2 n}$, namely,

$$
K_{n}=\left(\begin{array}{cc}
0 & I_{n} \\
I_{n} & 0
\end{array}\right)
$$


Set

$$
\begin{aligned}
& \mathfrak{h}(n)=\left\{X \in \mathfrak{g l}(2 n, \mathbb{R}) \mid X K_{n}-K_{n} X=0\right\}, \\
& s(n)=\left\{X \in \mathfrak{g l}(2 n, \mathbb{R}) \mid X K_{n}+K_{n} X=0\right\} .
\end{aligned}
$$

Then $\mathfrak{g} \mathfrak{l}(2 n, \mathbb{R})=\mathfrak{h}(n) \oplus s(n)$ and there are defined two projections

$$
\begin{aligned}
& R: \operatorname{gl}(2 n, \mathbb{R}) \rightarrow \mathfrak{h}(n) X \mapsto \frac{1}{2}\left(X+K_{n} X K_{n}\right), \\
& S: \operatorname{gl}(2 n, \mathbb{R}) \rightarrow s(n) X \mapsto \frac{1}{2}\left(X-K_{n} X K_{n}\right) .
\end{aligned}
$$

We have the following

Proposition 3.1. There exists a neighborhood $U$ of $K_{n}$ in $\mathcal{X}(n)$ such that every $P \in U$ can be written in a unique way as

$$
P=(I+L) K_{n}(I+L)^{-1},
$$

where $L K_{n}+K_{n} L=0$ and $\operatorname{det}(I+L) \neq 0$.

Proof. First of all, we observe that $\operatorname{GL}(2 n, \mathbb{R})$ acts transitively on the space $\mathcal{X}(n)$ of paracomplex structures on $\mathbb{R}^{2 n}$ by the following:

$$
P \mapsto A P A^{-1}
$$

for every $P \in \mathcal{X}(n)$ and $A \in \operatorname{GL}(2 n, \mathbb{R})$. The isotropy subgroup at $K_{n}$ is given by

$$
H(n)=\left\{A \in \mathrm{GL}(2 n, \mathbb{R}) \mid A K_{n}-K_{n} A=0\right\} .
$$

Therefore, $\mathcal{X}(n)=\operatorname{GL}(2 n, R) / H(n)$. Consequently, $\operatorname{GL}(2 n, \mathbb{R}) \stackrel{\pi}{\longrightarrow} \mathcal{X}(n)$ is an $H(n)$-principal bundle and $\pi(A)=A K_{n} A^{-1}$. By the local triviality, there exists a local section $\sigma: U \rightarrow \operatorname{GL}(2 n, R)$ such that

$$
\sigma\left(K_{n}\right)=I, \quad \sigma(P) K_{n} \sigma(P)^{-1}=P \quad \text { for all } P \in U .
$$

By definition of the projection $R$, we immediately have that $R\left(\sigma\left(K_{n}\right)\right)=I$; hence, if $U$ is small enough, $R(\sigma(P)) \in H(n)$, for every $P \in U$. Therefore,

$$
\hat{\sigma}(P)=\sigma(P)(R(\sigma(P)))^{-1}
$$

is a section over $U$ with $R(\hat{\sigma}(P)) \equiv I$. The section $\hat{\sigma}$ is determined by the two conditions

$$
\hat{\sigma}\left(K_{n}\right)=I, \quad R(\hat{\sigma}(P)) \equiv I .
$$

Therefore, for every paracomplex structure $P$, we have

$$
\begin{aligned}
P & =(R(\hat{\sigma}(P))+S(\hat{\sigma}(P))) K_{n}(R(\hat{\sigma}(P))+S(\hat{\sigma}(P)))^{-1} \\
& =(I+S(\hat{\sigma}(P))) K_{n}(I+S(\hat{\sigma}(P)))^{-1} .
\end{aligned}
$$


Setting

$$
S(\hat{\sigma}(P))=L,
$$

we obtain that $P$ can be written in a unique way as

$$
P=(I+L) K_{n}(I+L)^{-1},
$$

where $L K_{n}+K_{n} L=0$.

Let $K_{t}$, for $-\varepsilon<t<\varepsilon$, be a curve of almost paracomplex structures on $M$ such that $K_{0}=K$. In view of Proposition 3.1, $K_{t}$ can be uniquely written as

$$
K_{t}=\left(I+L_{t}\right) K\left(I+L_{t}\right)^{-1},
$$

where

$$
L_{t}=t L+o(t) \quad \text { and } \quad L_{t} K+K L_{t}=0 .
$$

Note that

$$
\left.\frac{d}{d t} K_{t}\right|_{t=0}=2 L K
$$

Indeed, by definition,

$$
\left.\frac{d}{d t} K_{t}\right|_{t=0}=\left.\frac{d}{d t}\left(I+L_{t}\right) K\left(I+L_{t}\right)^{-1}\right|_{t=0}=L K-K L=2 L K .
$$

The next proposition allows us to give an obstruction to the integrability of a curve of almost paracomplex structures.

Proposition 3.2. Let $K_{t}$ be a curve of almost paracomplex structures, defined for $-\varepsilon<t<\varepsilon$, such that $K_{0}=K$. Then

$$
\left.\frac{d}{d t} N_{K_{t}}(X, Y)\right|_{t=0}=4\left(\bar{\partial}_{K} L\right)(X, Y)-N_{K}(L X, Y)-N_{K}(X, L Y) .
$$

Proof. Let

$$
K_{t}=\left(I+L_{t}\right) K\left(I+L_{t}\right)^{-1},
$$

where

$$
L_{t}=t L+o(t) \quad \text { and } \quad L_{t} K+K L_{t}=0 .
$$

Then, setting $\dot{K}_{0}=\left.\frac{d}{d t} K_{t}\right|_{t=0}$, we obtain

$$
\begin{aligned}
\left.\frac{d}{d t} N_{K_{t}}(X, Y)\right|_{t=0}= & {\left[\dot{K}_{0} X, K Y\right]+\left[K X, \dot{K}_{0} Y\right]-\dot{K}_{0}[K X, Y] } \\
& -K\left[\dot{K}_{0} X, Y\right]-\dot{K}_{0}[X, K Y]-K\left[X, \dot{K}_{0} Y\right]
\end{aligned}
$$


Since $\dot{K}_{0}=2 L K$, we get

$$
\begin{aligned}
\left.\frac{d}{d t} N_{K_{t}}(X, Y)\right|_{t=0} \\
=2\{-[K L X, K Y]-[K X, K L Y]+K L[K X, Y] \\
\quad+K[K L X, Y]+K L[X, K Y]+K[X, K L Y]\} \\
=2\{K L[K X, Y]+K L[X, K Y]+[L X, Y]+[X, L Y] \\
\left.\quad-K[L X, K Y]-K[K X, L Y]-N_{K}(L X, Y)-N_{K}(X, L Y)\right\} .
\end{aligned}
$$

On the other hand, a direct computation gives

$$
\begin{aligned}
4\left(\bar{\partial}_{K} L\right)(X, Y)=2[ & X, L Y]-2 K[K X, L Y]+N_{K}(L Y, X) \\
& -2[Y, L X]+2 K[K Y, L X]-N_{K}(L X, Y) \\
& +2 K L[K X, Y]+2 K L[X, K Y] .
\end{aligned}
$$

The proof of the proposition follows from the last two equalities.

As a direct consequence, we have the following

Corollary 3.3. If $K_{t}$ is a curve of paracomplex structures, then $\bar{\partial}_{K} L=0$.

\section{Deformations of paracomplex structures}

Let $M$ be a compact manifold endowed with a paracomplex structure $K$. We are going to characterize small deformations of $K \in \Gamma(M$, End $(T M))$, using the DGLA $\left(\mathcal{A},[[]],, \bar{\partial}_{K}\right)$ introduced in Section 5.

On the space $\Gamma(M, \operatorname{End}(T M))$ of $\bigodot^{\infty}$-sections of the vector bundle $\operatorname{End}(T M)=$ $T M^{*} \otimes T M$ we will consider the usual topology.

In view of Proposition 3.1, every almost paracomplex structure $\widehat{K}$ close to $K$ can be written in a unique way as

$$
\widehat{K}=(I+L) K(I+L)^{-1},
$$

with $L K+K L=0, \operatorname{det}(I+L) \neq 0$.

The following proposition describes the behavior of the Nijenhuis tensor of the deformed almost paracomplex structure $\widehat{K}$.

Proposition 4.1. Let $K$ be a paracomplex structure on a manifold $M$ and

$$
\widehat{K}=(I+L) K(I+L)^{-1},
$$

with $L K+K L=0, \operatorname{det}(I+L) \neq 0$. Then

$$
(I+L)^{-1} N_{\widehat{K}}((I+L) X,(I+L) Y)=4\left(I-L^{2}\right)^{-1}\left(\bar{\partial}_{K} L+\frac{1}{2}[[L, L]]\right)(X, Y) .
$$


Proof. The formula to prove is equivalent to

$$
(I-L) N_{\widehat{K}}((I+L) X,(I+L) Y)=4\left(\bar{\partial}_{K} L+\frac{1}{2}[[L, L]]\right)(X, Y) .
$$

A straightforward computation, taking into account the integrability condition $N_{K}=$ 0 and $L K+K L=0$, gives

$$
\begin{aligned}
(I-L) N_{\widehat{K}} & ((I+L) X,(I+L) Y) \\
=[ & K, L K Y]+[L K X, K Y]+[L K X, L K Y] \\
& +[X, L Y]+[L X, Y]+[L X, L Y] \\
& -K([K X, L Y]+[L K X, Y]+[L K X, L Y]) \\
& -K([X, L K Y]+[L X, K Y]+[L X, L K Y]) \\
& -L([K X, K Y]+[K X, L K Y]+[L K X, K Y]) \\
& -L([X, Y]+[X, L Y]+[L X, Y]) \\
& +K L([K X, Y]+[K X, L Y]+[L K X, Y]) \\
& +K L([X, K Y]+[X, L K Y]+[L X, K Y]) \\
=4 & ([X, L Y]-K[K X, L Y]-[Y, L X]+K[K Y, L X]) \\
& -2(L[X, Y]+[K X, K Y]-[L X, L Y]-[K L X, K L Y]) \\
& -L[L X, Y]+L[K L X, K Y]-K L[K L X, Y]+K L[L X, K Y] \\
& -L[X, L Y]+L[K X, K L Y]-K L[X, K L Y]+K L[K X, L Y] \\
=4 & \bar{\partial}_{K} L(X, Y)+4[[L X, L Y]]+2 L\left(\left(\bar{\partial}_{K} X\right)(L Y)-\left(\bar{\partial}_{K} Y\right)(L X)\right) \\
& +2 L\left(\left(\bar{\partial}_{K} K Y\right)(K L X)-\left(\bar{\partial}_{K} K X\right)(K L Y)\right) \\
=4 & \left(\bar{\partial}_{K} L+\frac{1}{2}[[L, L]]\right)(X, Y),
\end{aligned}
$$

where in the last equality we have used formula (2).

Therefore, by Propositions 3.1 and 4.1, we obtain the following

Theorem 4.2. Let $(M, K)$ be a compact paracomplex manifold. Then the map between

$$
\left\{L \in \Gamma(M, \operatorname{End}(T M)) \mid L K+K L=0, \bar{\partial}_{K} L+\frac{1}{2}[[L, L]]=0, \operatorname{det}(I+L) \neq 0\right\}
$$

and

$$
\left\{\widehat{K} \in \Gamma(M, \operatorname{End}(T M)) \mid \widehat{K}^{2}=I, N_{\widehat{K}}=0\right\},
$$

given by

$$
L \mapsto \widehat{K}=(I+L) K(I+L)^{-1},
$$

is a bijection between a neighborhood of $0 \in \Gamma(M, \operatorname{End}(T M))$ and a neighborhood of $K$. 
As an example, we consider the standard $2 n$-dimensional torus $\mathbb{T}^{2 n}=\mathbb{R}^{2 n} / \mathbb{Z}^{2 n}$ endowed with the standard paracomplex structure defined by

$$
K_{n}\left(\frac{\partial}{\partial x_{j}}\right)=\frac{\partial}{\partial y_{j}}, \quad K_{n}\left(\frac{\partial}{\partial y_{j}}\right)=\frac{\partial}{\partial x_{j}}, \quad j=1, \ldots, n .
$$

Let $K_{t}$ be a curve of paracomplex structures on $\mathbb{\square}^{2 n}$ such that $K_{0}=K_{n}$, namely

$$
K_{t}=\left(I+L_{t}\right) K_{n}\left(I+L_{t}\right)^{-1}
$$

Then the tangent vector $V=\dot{K}_{0}$ is a constant endomorphism of $T\left(\mathbb{T}^{2 n}\right)$ which anticommutes with $K_{n}$. Conversely, starting with such a constant $V \in \operatorname{End}\left(T\left(\mathbb{T}^{2 n}\right)\right)$ and setting $L=\frac{1}{2} V K_{n}$, we construct a curve of almost paracomplex structures given by

$$
K_{t}=(I+t L) K_{n}(I+t L)^{-1},
$$

which is in fact integrable since

$$
\bar{\partial}_{K_{n}}(t L)+\frac{1}{2} t^{2}[[L, L]]=0
$$

\section{Families of paracomplex structures on 4-dimensional nilmanifolds}

In this section we consider 4-dimensional nilmanifolds endowed with a fixed paracomplex structure and we compute the invariant small paracomplex deformations of it.

It is well known that there are only two real 4-dimensional nilpotent non-abelian Lie algebras. According to the Malcev Theorem (see [12]) it turns out that the simplyconnected Lie groups associated with these two Lie algebras have compact quotients.

5.1. Four-dimensional nilmanifolds with $\boldsymbol{b}_{\mathbf{1}}=\mathbf{3}$. Let $\mathfrak{h}(3)$ be the 3-dimensional real Heisenberg Lie algebra and $\mathfrak{n}_{1}=\mathfrak{h}(3) \oplus \mathbb{R}$. Then we can find a basis $\left\{f^{1}, \ldots, f^{4}\right\}$ of $n_{1}^{*}$ such that

$$
d f^{1}=0, \quad d f^{2}=-f^{1} \wedge f^{3}, \quad d f^{3}=0, \quad d f^{4}=0 .
$$

Therefore, denoting by $\left\{f_{1}, \ldots, f_{4}\right\}$ the dual basis of $\left\{f^{1}, \ldots, f^{4}\right\}$, we obtain that the only non-zero bracket is $\left[f_{1}, f_{3}\right]=f_{2}$.

Let $N_{1}=H(3) \times \mathbb{R}$ be the simply-connected Lie group with Lie algebra $\mathfrak{n}$, where

$$
H(3)=\left\{A=\left(\begin{array}{lll}
1 & x & z \\
0 & 1 & y \\
0 & 0 & 1
\end{array}\right) \mid x, y, z, \in \mathbb{R}\right\}
$$

is the real 3-dimensional Heisenberg group, and let $M=\Gamma \backslash N_{1}$ be any compact quotient of $N_{1}$. As an example, we can take a primary Kodaira-Thurston surface 
$\Gamma \backslash H(3) \times \mathbb{\$}^{1}$, where $\Gamma \subset H(3)$ is the subgroup of $H(3)$ given by the matrices with integer entries.

Let $K \in \operatorname{End}(T M)$ be the paracomplex structure defined as

$$
K f_{1}=f_{3}, \quad K f_{2}=f_{4}, \quad K f_{3}=f_{1}, \quad K f_{4}=f_{2} .
$$

Then, setting $\boldsymbol{t}=\left(t_{1}, \ldots, t_{8}\right) \in \mathbb{R}^{8}$, every invariant endomorphism of $T M$ anticommuting with $K$ has the form

$$
L_{\boldsymbol{t}}=\left(\begin{array}{rrrr}
t_{1} & t_{2} & t_{3} & t_{4} \\
t_{5} & t_{6} & t_{7} & t_{8} \\
-t_{3} & -t_{4} & -t_{1} & -t_{2} \\
-t_{7} & -t_{8} & -t_{5} & -t_{6}
\end{array}\right) .
$$

A straightforward computation yields

$$
\left(\bar{\partial}_{K} L_{\boldsymbol{t}}\right)\left(f_{1}, f_{2}\right)=\frac{1}{2}\left(-t_{4} f_{2}+t_{2} f_{4}\right)
$$

and

$$
\left[\left[L_{\boldsymbol{t}}, L_{\boldsymbol{t}}\right]\right]\left(f_{1}, f_{2}\right)=\frac{1}{2}\left\{2 t_{2} t_{4} f_{1}+\left(t_{2} t_{8}+t_{4} t_{6}\right) f_{2}-\left(t_{2}^{2}+t_{4}^{2}\right) f_{3}-\left(t_{2} t_{6}+t_{4} t_{8}\right) f_{4}\right\} .
$$

Therefore,

$$
\bar{\partial}_{K} L_{\boldsymbol{t}}+\frac{1}{2}\left[\left[L_{\boldsymbol{t}}, L_{\boldsymbol{t}}\right]\right]=0
$$

if and only if

$$
t_{2}=t_{4}=0 .
$$

Hence, for such $L_{\boldsymbol{t}}$ satisfying the above conditions and $|\boldsymbol{t}|<\varepsilon$, it follows that $K_{\boldsymbol{t}}=\left(I+L_{\boldsymbol{t}}\right) K\left(I+L_{\boldsymbol{t}}\right)^{-1}$ gives rise to a 6-parameter family of paracomplex structures on $M$, with $K_{0}=K$.

Remark 5.1. Consider the pseudo-Riemannian metric on $M$ defined by

$$
g=-f^{1} \odot f^{4}+f^{2} \odot f^{3} .
$$

Then $g$ is a para-Hermitian metric, whose fundamental form

$$
\omega=-f^{1} \wedge f^{2}+f^{3} \wedge f^{4}
$$

is $d$-closed, so that $g$ is a para-Kähler metric on $(M, K)$. Let

$$
\omega_{t}=\frac{1+t_{8}^{2}-t_{6}^{2}}{t_{8}^{2}-\left(t_{6}+1\right)^{2}} f^{1} \wedge f^{2}+\frac{2 t_{8}}{t_{8}^{2}-\left(t_{6}+1\right)^{2}} f^{2} \wedge f^{3}+f^{3} \wedge f^{4} .
$$

A straightforward computation shows that, for

$$
t_{1}=t_{2}=t_{3}=t_{4}=0, \quad 1+t_{8}^{2}-t_{6}^{2}>0, \quad t_{8}^{2}-\left(t_{6}+1\right)^{2}<0,
$$


$\left(K_{t}, \omega_{t}\right)$ is a family of para-Kähler structures on $M$ such that $\left(K_{0}, \omega_{0}\right)=(K, \omega)$, where

$$
K_{t}=\left(\begin{array}{cccc}
0 & 0 & 1 & 0 \\
-2 \frac{t_{5} t_{8}-t_{7} t_{6}-t_{7}}{-t_{6}^{2}+1+t_{8}^{2}} & 2 \frac{t_{8}}{-t_{6}^{2}+1+t_{8}^{2}} & -2 \frac{-t_{5} t_{6}-t_{5}+t_{7} t_{8}}{-t_{6}^{2}+1+t_{8}^{2}} & -\frac{t_{8}^{2}-t_{6}^{2}-2 t_{6}-1}{-t_{6}^{2}+1+t_{8}^{2}} \\
1 & 0 & 0 & 0 \\
-2 \frac{-t_{5} t_{6}+t_{5}+t_{7} t_{8}}{-t_{6}^{2}+1+t_{8}^{2}} & -\frac{-t_{6}^{2}+2 t_{6}-1+t_{8}^{2}}{-t_{6}^{2}+1+t_{8}^{2}} & -2 \frac{-t_{7} t_{6}+t_{7}+t_{5} t_{8}}{-t_{6}^{2}+1+t_{8}^{2}} & -2 \frac{t_{8}}{-t_{6}^{2}+1+t_{8}^{2}}
\end{array}\right) .
$$

5.2. Four-dimensional nilmanifolds with $\boldsymbol{b}_{1}=2$. Let $\mathfrak{n}_{2}$ be the 4-dimensional real Lie algebra whose dual space is spanned by $\left\{e^{1}, \ldots, e^{4}\right\}$ satisfying the following Cartan structure equations:

$$
d e^{1}=0, \quad d e^{2}=0, \quad d e^{3}=e^{1} \wedge e^{2}, \quad d e^{4}=e^{1} \wedge e^{3} .
$$

Denoting by $\left\{e_{1}, \ldots, e_{4}\right\}$ the dual basis of $\left\{e^{1}, \ldots, e^{4}\right\}$, we obtain

$$
\left[e_{1}, e_{2}\right]=-e_{3}, \quad\left[e_{1}, e_{3}\right]=-e_{4} .
$$

Let $N_{2}$ be the simply-connected Lie group having Lie algebra g. Let $M=\Gamma \backslash N_{2}$ be any compact quotient of $N_{2}$, i.e., $M$ is a compact 4-dimensional nilmanifold. Define an $N_{2}$-invariant almost paracomplex structure $K$ on $M$, by setting

$$
K e_{1}=e_{1}, \quad K e_{2}=-e_{2}, \quad K e_{3}=-e_{3}, \quad K e_{4}=e_{4}
$$

Then, a direct computation shows that $N_{K}=0$, i.e., $K$ gives rise to a paracomplex structure on $M$. Now, using Theorem 4.2, we are going to compute the small deformations of $K$ corresponding to invariant endomorphisms $L$ of $T M$, which anticommute with $K$.

In order to perform the computation, it will be useful to make a change of basis of forms, namely, we set

$$
f^{1}=e^{1}+e^{2}, \quad f^{2}=e^{3}+e^{4}, \quad f^{3}=e^{1}-e^{2}, \quad f^{4}=-e^{3}+e^{4},
$$

with dual basis

$$
f_{1}=\frac{1}{2}\left(e_{1}+e_{2}\right), \quad f_{2}=\frac{1}{2}\left(e_{3}+e_{4}\right), \quad f_{3}=\frac{1}{2}\left(e_{1}-e_{2}\right), \quad f_{4}=\frac{1}{2}\left(-e_{3}+e_{4}\right) \text {. }
$$

Then, we have

$$
K f_{1}=f_{3}, \quad K f_{2}=f_{4}, \quad K f_{3}=f_{1}, \quad K f_{4}=f_{2} .
$$

Setting $\boldsymbol{t}=\left(t_{1}, \ldots, t_{8}\right) \in \mathbb{R}^{8}$, any invariant endomorphism of $T M$ anticommuting with $K$ is represented with respect to the basis $\left\{f_{1}, \ldots, f_{4}\right\}$ by the matrix

$$
L_{t}=\left(\begin{array}{rrrr}
t_{1} & t_{2} & t_{3} & t_{4} \\
t_{5} & t_{6} & t_{7} & t_{8} \\
-t_{3} & -t_{4} & -t_{1} & -t_{2} \\
-t_{7} & -t_{8} & -t_{5} & -t_{6}
\end{array}\right)
$$


A tedious computation shows that the Maurer-Cartan equation

$$
\bar{\partial}_{K} L_{t}+\frac{1}{2}\left[\left[L_{t}, L_{t}\right]\right]=0
$$

holds if and only if

$$
t_{1}=t_{3}, \quad t_{2}=t_{4}=0, \quad t_{6}=-t_{8}
$$

In such a case,

$$
K_{\boldsymbol{t}}=\left(I+L_{\boldsymbol{t}}\right) K\left(I+L_{\boldsymbol{t}}\right)^{-1}
$$

is a 4-parameter family of paracomplex structures on $M$, with $K_{0}=K$.

Remark 5.2. It has to be noted that any compact quotient $M$ of $N_{2}$ has no complex structures for cohomological reasons (see e.g. [8]). Indeed, by Nomizu's Theorem (see [14]), we have that $b_{1}(M)=2$. Furthermore, by a result of Kodaira (see [10], Theorem 25), a complex surface is a deformation of an algebraic surface if and only if its first Betti number is even. Therefore, if $J$ were a complex structure on $M$, then $(M, J)$ should have a Kähler metric. But this is not possible since, by the theorem of Benson and Gordon (see [2]), if $M$ is a compact nilmanifold carrying a Kähler structure, then $M$ is diffeomorphic to a torus.

5.3. Four-dimensional tori. According to $\S 4$, any matrix $L_{\boldsymbol{t}}$ as in (3), with $|\boldsymbol{t}|<\varepsilon$, gives rise to a paracomplex structure on $\mathbb{T}^{4}$.

\section{References}

[1] D. V. Alekseevskiŭ, K. Medori, and A. Tomassini, Homogeneous para-Kählerian Einstein manifolds. Uspekhi Mat. Nauk 64 (2009), no. 1, 3-50; English transl. Russian Math. Surveys 64 (2009), 1-43. Zbl 1179.53050 MR 2503094

[2] C. Benson and C. S. Gordon, Kähler and symplectic structures on nilmanifolds. Topology 27 (1988), 513-518. Zbl 0672.53036 MR 976592

[3] R. L. Bryant, Bochner-Kähler metrics. J. Amer. Math. Soc. 14 (2001), 623-715. Zbl 1006.53019 MR 1824987

[4] S. Console and A. Fino, Dolbeault cohomology of compact nilmanifolds. Transform. Groups 6 (2001), 111-124. Zbl 1028.58024 MR 1835667

[5] V. Cortés, C. Mayer, T. Mohaupt, and F. Saueressig, Special geometry of Euclidean supersymmetry 1. Vector multiplets. J. High Energy Phys. 03 (2004), 028. Zbl MR 2061551

[6] V. Cruceanu, P. Fortuny, and P. M. Gadea, A survey on paracomplex geometry. Rocky Mountain J. Math. 26 (1996), 83-115. Zbl 0856.53049 MR 1386154

[7] P. de Bartolomeis and F. Meylan, Intrinsic deformation theory of CR structures. Ann. Sc. Norm. Super. Pisa Cl. Sci. (5) 9 (2010), 459-494. Zbl 1206.32015 MR 2722651 
[8] M. Fernández, M. J. Gotay, and A. Gray, Compact parallelizable four-dimensional symplectic and complex manifolds. Proc. Amer. Math. Soc. 103 (1988), 1209-1212. Zbl 0656.53034 MR 955011

[9] K. Fukaya, Deformation theory, homological algebra and mirror symmetry. In Geometry and physics of branes (Como, 2001), Ser. High Energy Phys. Cosmol. Gravit., IOP, Bristol 2003, 121-209. MR 1950958

[10] K. Kodaira, On the structure of compact complex analytic surfaces, I. Amer. J. Math. 86 (1964), 751-798. Zbl 0137.17501 MR 0187255

[11] P. Libermann, Sur les structures presque paracomplexes. C. R. Acad. Sci. Paris 234 (1952), 2517-2519. Zbl 0046.15601 MR 0048893

[12] A. I. Malcev, On a class of homogeneous spaces. Izv. Akad. Nauk SSSR Ser. Mat. 13 (1949), 9-32; Amer. Math. Soc. Transl. (1) 9, Amer. Math. Soc., Providence, RI, 1962, 276-307. Zbl 0034.01701 MR 0039734

[13] J. Morrow and K. Kodaira, Complex manifolds. AMS Chelsea Publishing, Providence, RI, 2006. Zbl 1087.32501 MR 2214741

[14] K. Nomizu, On the cohomology of compact homogeneous spaces of nilpotent Lie groups. Ann. of Math. (2) 59 (1954), 531-538. Zbl 0058.02202 MR 0064057

[15] S. Rollenske, Lie-algebra Dolbeault-cohomology and small deformations of nilmanifolds. J. London Math. Soc. (2) 79 (2009), 346-362. Zbl 1194.32006 MR 2496518

Received March 15, 2010

C. Medori, Dipartimento di Matematica, Università di Parma, Parco Area delle Scienze, 53/A, 43124 Parma, Italy

E-mail: costantino.medori@unipr.it

A. Tomassini, Dipartimento di Matematica, Università di Parma, Parco Area delle Scienze, 53/A, 43124 Parma, Italy

E-mail: adriano.tomassini@unipr.it 